\title{
Antibacterial and antibiotic-modifying activities of three food plants (Xanthosoma mafaffa Lam., Moringa oleifera (L.) Schott and Passiflora edulis Sims) against multidrug-resistant (MDR) Gram-negative bacteria
}

Joachim K. Dzotam, Francesco K. Touani and Victor Kuete

\begin{abstract}
Background: The present study was designed to investigate the antibacterial activities of the methanol extract of three edible plants, namely Xanthosoma mafaffa, Moringa oleifera and Passiflora edulis and their synergistic effects with some commonly used antibiotics against MDR Gram-negative bacteria expressing active efflux pumps.

Methods: Broth microdilution method was used to determine the minimum inhibitory concentrations (MICS) and the minimum bactericidal concentrations (MBCs) of the extracts, as well as those of antibiotics in association with the extracts.

Results: The phytochemical test indicate that all tested crude extracts contained polyphenols, triterpenes and steroids whilst other phytochemical classes were selectively distributed. Extracts showed antibacterial activities with minimum inhibitory concentrations ranging from 128-1024 $\mathrm{gg} / \mathrm{mL}$ on the majority of the 19 tested Gram-negative bacterial strains. Extract from the pericarp of $P$. edulis inhibited the growth of $89.5 \%$ of the 19 tested bacterial strains, the lowest minimal inhibitory concentration (MIC) value of $128 \mu \mathrm{g} / \mathrm{mL}$ being recorded against Escherichia coli AG100 strain. In the presence of Phenylalanine-Arginine $\beta$-Naphtylamide (PABN)], an efflux pump inhibitor (EPI), the activity of the extract from $X$. mafaffa increased on $40 \%$ of tested strains. In combination with antibiotics, extracts of $X$. mafaffa, $M$. oleifera and pericarp of $P$. edulis showed synergistic effects with some antibiotics against more than $75 \%$ of the tested bacteria.
\end{abstract}

Conclusion: The results of the present study indicate that the tested plants may be used in the treatment of bacterial infections including the multi-resistant bacteria.

Keywords: Antibiotic-potentiation, Gram-negative bacteria, Moringa oleifera, Multi-resistance, Passiflora edulis, Xanthosoma mafaffa

* Correspondence: kuetevictor@yahoo.fr

Department of Biochemistry, Faculty of Science, University of Dschang, P.O.

Box 67, Dschang, Cameroon 


\section{Background}

Infectious diseases remain the major cause of mortality amongst children and young adults worldwide, with higher prevalence in developing countries [1]. Despite the abundance of antibiotics used in chemotherapy, there is a drastic increase of resistant bacteria. Resistance to antibiotics occurs typically as a result of drug inactivation or modification, target alteration, or reduced accumulation associated with decreased permeability and or increased efflux [2]. The scarcity of new antimicrobials active against MDR bacteria propels the search of chemotherapeutic agents. While 25-50\% of current pharmaceuticals are derived from natural products, it was reported that none are used as antimicrobials [3]. Investigation of substances which can potentiate the activity of commonly used antibiotics are also being intensified [4-7]. Previous studies documented the good antimicrobial potential of natural products from higher plants $[8,9]$. Several food plants were also documented as potential candidate to fight MDR Gram-negative bacteria. Some of them include Dichrostachys glomerata, Beilschmiedia cinnamomea, Aframomum citratum, Piper capense, Echinops giganteus, Fagara xanthoxyloïdes and Olax subscorpioïdea [4], Lactuca sativa, Sechium edule, Cucurbita pepo and Solanum nigrum [10], Piper nigrum and Vernonia amygdalina [11], Beilschmiedia obscura, Pachypodanthium staudtii and Peperomia fernandopoiana [12], Capsicum frutescens [13]. In our continous search of functional food plants, we designed the present work to investigate in vitro antibacterial activity of the methanol extracts of three Cameroonian food plants, Moringa oleifera Lam. (Moringaceae), Xanthosoma mafaffa (L.) Schott (Araceae), Passiflora edulis Sims (Passifloraceae) against MDR bacteria. The study was extended to the ability of the studied extracts to potentiate the activity of some commonly used antibiotics against some of the tested MDR bacteria.

\section{Methods \\ Plant material and extraction}

The three food plants used in this work were purchased from the Bafoussam markets (West Region of Cameroon) in January 2014. The collected plant material were the leaves of Xanthosoma mafaffa, Moringa oleifera and the fruits of Passiflora edulis. The plants were identified at the National herbarium (Yaounde, Cameroon) where voucher specimens were deposited under the reference numbers (Table 1). Each plant sample was air dried and then powdered. The obtained powder (200 g) was extracted with methanol $(\mathrm{MeOH} ; 1 \mathrm{~L})$ for $48 \mathrm{~h}$ at room temperature with momentary shaking. Methanol was then removed under reduced pressure to give residues which constituted the crude extract. All extracts were then kept at $4{ }^{\circ} \mathrm{C}$ until further use.

\section{Preliminary phytochemical screening}

The major phytochemical classes such as alkaloids (Dragendorff's and Mayer's tests), triterpenes (Libermann Burchard's test), flavonoids (Aluminum chloride test), anthraquinones (Borntrager's test), polyphenols (Ferric chloride test), sterols (Salkowski's test), coumarins (Lacton test), saponins (Foam test) and tannins (Gelatin test) (Table 2) were investigated according to the commonly described phytochemical methods [14-17].

\section{Antimicrobial assays}

\section{Chemicals for antimicrobial assay}

Tetracycline (TET), cefepime (CEP), ciprofloxacin (CIP), norfloxacin (NOR), chloramphenicol (CHL), ampicillin (AMP), erythromycin (ERY), kanamycin (KAN) (Sigma-

Table 1 Information on plants used in the present study

\begin{tabular}{|c|c|c|c|c|}
\hline $\begin{array}{l}\text { Species (family); } \\
\text { Voucher number }^{a}\end{array}$ & Traditional uses & $\begin{array}{l}\text { Parts used } \\
\text { traditionally }\end{array}$ & $\begin{array}{l}\text { Bioactive or potentially } \\
\text { bioactive components }\end{array}$ & $\begin{array}{l}\text { Bioactivity of crude } \\
\text { extract }\end{array}$ \\
\hline $\begin{array}{l}\text { Xanthosoma mafaffa } \\
\text { (L.) Schott (Araceae); } \\
\text { 18675/SRF/Cam }\end{array}$ & $\begin{array}{l}\text { Bone disease } \\
\text { (osteoporosis) [37] }\end{array}$ & $\begin{array}{l}\text { Leaves and } \\
\text { tubers }\end{array}$ & - & - \\
\hline $\begin{array}{l}\text { Passiflora edulis } \\
\text { Sims (Passifloraceae); } \\
65104 / \text { HNC }\end{array}$ & $\begin{array}{l}\text { Anxiety, insomnia and } \\
\text { nervousnes, antifungal, } \\
\text { anti-inflammatory, } \\
\text { antihypertensive [38], } \\
\text { gastric trouble [39], } \\
\text { antioxidant, cancer [40] }\end{array}$ & $\begin{array}{l}\text { Leaves, fruit, bark } \\
\text { and roots }\end{array}$ & $\begin{array}{l}\text { ionone-I, ionone-II, megastigma-5,8-dien-4-1, } \\
\text { megastigma-5,8(Z)-diene-4-1, 4,4a-Epoxy-4, } \\
\text { 4a-dihydroedulan, 3-hydroxyedulan, edulan-I, } \\
\text { edulan-II, passifloric acid methyl ester [40] }\end{array}$ & $\begin{array}{l}\text { Methanol extract : Sa, Sf, } \\
\text { BS, EC, PV and St [40] }\end{array}$ \\
\hline $\begin{array}{l}\text { Moringa oleifera } \\
\text { Lam. (Moringaceae); } \\
49178 / \text { HNC }\end{array}$ & $\begin{array}{l}\text { Dental caries, syphilis, } \\
\text { typhoid, diarrhea, } \\
\text { epilepsy, purgative, } \\
\text { prostate cancer, water } \\
\text { purification [41], fever, } \\
\text { HIV-AIDS [42] }\end{array}$ & $\begin{array}{l}\text { Leaves, flowers, } \\
\text { seeds and barks }\end{array}$ & $\begin{array}{l}\text { 4-(4'-O-acetyl-a-L-rhamnopyranosyloxy) } \\
\text { benzylisothiocyanate, } 4-(-L \text {-rhamnopyranosyloxy) } \\
\text { benzylisothiocyanate, niazimicin, pterygospermin, } \\
\text { benzylisothiocyanate and 4-( } a \text {-L-rhamnopyranosyloxy) } \\
\text { benzylglucosinolate [43] }\end{array}$ & $\begin{array}{l}\text { Aqueous and ethanol } \\
\text { extracts of seeds against } \\
\text { Sa, Vc, Ec, Se, LV and On }\end{array}$ \\
\hline
\end{tabular}

${ }^{a}$ (HNC) Herbier National du Cameroun, (SRF/Cam) Société des Réserves Forestières du Cameroun; (-): nor reported; Sa: Staphylococcus aureus; Vc: Vibrio cholerae; Ec: Escherichia coli; Se: Salmonella enteretidis; Lv: Litopenaeus vannmaei; On: Oreochromis nicoticus; Bs: Bacillus subtilis; St: Salmonella typhi; Sf: Streptococcus faecalis; Pv: Proteus vulgaris; HIV-AIDS: Human Immunodeficiency Virus- Acquired Immuno Deficiency Syndrome 
Table 2 Extraction yields and phytochemical composition of the plant extracts

\begin{tabular}{llll}
\hline Extracts & $\begin{array}{l}\text { Xanthosoma mafaffa } \\
\text { leaves extact }\end{array}$ & $\begin{array}{l}\text { Passiflora edulis } \\
\text { Pericarps (fruits) } \\
\text { extract }\end{array}$ & $\begin{array}{l}\text { Moringa oleifera } \\
\text { leaves extract }\end{array}$ \\
\hline Yield ${ }^{\mathrm{a}}(\%)$ & 4.30 & 3.92 & 3.95 \\
Alkaloids & - & - & + \\
Polyphenols & + & + & + \\
Flavonoids & - & + & + \\
Anthraquinones & - & - & + \\
Coumarins & + & - & + \\
Tannins & + & - & + \\
Triterpenes & + & + & + \\
Sterols & + & + & + \\
Saponins & + & + & + \\
\hline
\end{tabular}

$(-)$ : Absent; (+): Present; ${ }^{\text {a } y i e l d ~ c a l c u l a t e d ~ a s ~ t h e ~ r a t i o ~ o f ~ t h e ~ m a s s ~ o f ~ t h e ~}$ obtained methanol extract/mass of the plant powder

Aldrich, St Quentin Fallavier, France) were used as reference antibiotics (RA). $p$-Iodonitrotetrazolium chloride (INT; Sigma-Aldrich) and Phenylalanine-Arginine-ßNaphthylamide (PAßN; Sigma-Aldrich) were used as microbial growth indicator and efflux pumps inhibitor (EPI) respectively $[18,19]$.

\section{Microbial strains and culture media}

The studied microorganisms included sensitive and resistant strains of Escherichia coli (ATTC8739, AG100, AG100A, AG102, AG100ATet, W3110), Enterobacter aerogenes (ATCC13048, EA289, EA27, EA298, CM64), Klebsiella pneumoniae (ATCC11296, KP55, KР63, K24), Pseudomonas aeruginosa (PA01, PA124), Providencia stuartii (ATCC29914, NEA16) obtained clinically or from the American Type Culture Collection (ATCC). Their resistance profiles have been previously reported $[7,13,20]$. Nutrient agar were used for the activation of the tested Gram-negative bacteria [21].

\section{INT colorimetric assay for MIC and MBC determinations}

The MIC determinations on the tested bacteria were conducted using rapid $p$-iodonitrotetrazolium chloride (INT) colorimetric assay according to described methods [18] with some modifications [22, 23]. The test samples and RA were first of all dissolved in DMSO/Mueller Hinton Broth (MHB) or DMSO/7H9 broth. The final concentration of DMSO was lower than $2.5 \%$ and does not affect the microbial growth [24, 25]. The solution obtained was then added to Mueller Hinton Broth, and serially diluted two fold (in a 96- wells microplate). One hundred microlitre $(100 \mu \mathrm{L})$ of inoculum

Table 3 Minimal Inhibitory Concentration (MIC) in $\mu \mathrm{g} / \mathrm{mL}$ of methanol extracts from the studied plants and chloramphenicol

\begin{tabular}{|c|c|c|c|c|c|c|c|c|c|c|c|c|c|}
\hline \multirow[t]{3}{*}{ Bacterial strains } & & \multicolumn{12}{|c|}{ Tested samples, MIC and MBC and MIC in the presence of PABN in parenthesis $(\mu \mathrm{g} / \mathrm{mL})$} \\
\hline & & \multicolumn{3}{|c|}{ Xanthosoma mafaffa } & \multicolumn{3}{|c|}{ Passiflora edulis } & \multicolumn{3}{|c|}{ Moringa oleifera } & \multicolumn{3}{|c|}{ Chloramphenicol } \\
\hline & & MIC & $\mathrm{MBC}$ & R & $\mathrm{MIC}$ & $\mathrm{MBC}$ & $\mathrm{R}$ & MIC & MBC & $\mathrm{R}$ & MIC & MBC & R \\
\hline \multirow[t]{6}{*}{ E. coli } & ATCC8739 & - & - & - & 256 & - & - & 256 & - & - & 4 & - & - \\
\hline & AG100 & $256(512)$ & - & - & $128(<4)$ & 1024 & 8 & $128(256)$ & - & - & $4(<4)$ & 256 & 64 \\
\hline & AG100A & 512 & 1024 & 2 & 512 & 1024 & 2 & 512 & - & - & 2 & 64 & 32 \\
\hline & AG102 & $1024(512)$ & - & - & $512(1024)$ & 1024 & 2 & $256(1024)$ & - & - & $8(<4)$ & - & - \\
\hline & AG100ATet & $256(-)$ & - & - & $1024(-)$ & - & - & $1024(-)$ & 1024 & 1 & $64(32)$ & 256 & 4 \\
\hline & W3110 & 1024 & - & - & 256 & - & - & 256 & - & - & 8 & 16 & 2 \\
\hline \multirow[t]{5}{*}{ E. aerogenes } & ATCC13048 & - & - & - & 256 & - & - & 1024 & - & - & 8 & 128 & 16 \\
\hline & EA289 & $512(1024)$ & - & - & $512(1024)$ & 1024 & 2 & 1024 (1024) & - & - & $64(32)$ & 512 & 8 \\
\hline & EA27 & $256(16)$ & - & - & $256(16)$ & - & - & 1024 (1024) & - & - & $64(<4)$ & 512 & 8 \\
\hline & EA298 & - & - & - & 512 & - & - & - & - & - & 32 & 256 & 8 \\
\hline & CM64 & $-(-)$ & - & - & $-(-)$ & - & - & $-(-)$ & - & - & $256(4)$ & 256 & 1 \\
\hline \multirow[t]{4}{*}{ K. pneumoniae } & ATCC11296 & 256 & - & - & 256 & - & - & - & - & - & 4 & 512 & 128 \\
\hline & KP55 & $1024(512)$ & - & - & $512(1024)$ & - & - & 256 (1024) & - & - & $64(16)$ & 128 & 2 \\
\hline & KP63 & $1024(512)$ & - & - & $512(1024)$ & - & - & $-(-)$ & - & - & $256(32)$ & 256 & 1 \\
\hline & K24 & - & - & - & 1024 & - & - & - & - & - & 64 & 256 & 4 \\
\hline \multirow[t]{2}{*}{$P$. aeruginosa } & PA01 & - & - & - & 256 & - & - & 1024 & - & - & 16 & 256 & 16 \\
\hline & PA124 & $-(-)$ & - & - & $-(-)$ & - & - & $-(-)$ & - & - & $64(4)$ & 512 & 8 \\
\hline \multirow[t]{2}{*}{ P. stuartii } & ATCC29914 & - & - & - & 512 & - & - & 1024 & - & - & 8 & 32 & 4 \\
\hline & NEA16 & $512(1024)$ & - & - & $256(1024)$ & - & - & 1024 (1024) & - & - & $32(4)$ & 256 & 8 \\
\hline
\end{tabular}


$1.5 \times 10^{6} \mathrm{CFU} / \mathrm{mL}$ prepared in appropriate broth was then added [22, 23]. The plates were covered with a sterile plate sealer, then agitated to mix the contents of the wells using a plate shaker and incubated at $37{ }^{\circ} \mathrm{C}$ for $18 \mathrm{~h}$. The assay was repeated thrice. Wells containing adequate broth, $100 \mu \mathrm{L}$ of inoculum and DMSO to a final concentration of $2.5 \%$ served as negative control. The MIC of samples was detected after $18 \mathrm{~h}$ incubation at $37{ }^{\circ} \mathrm{C}$, following addition $(40 \mu \mathrm{L})$ of $0.2 \mathrm{mg} / \mathrm{mL}$ of INT and incubation at $37{ }^{\circ} \mathrm{C}$ for $30 \mathrm{~min}$. Viable bacteria reduced the yellow dye to a pink. MIC was defined as the lowest sample concentration that prevented the color change of the medium and exhibited complete inhibition of microbial growth [18]. The MBC was determined by adding $50 \mu \mathrm{L}$ aliquots of the preparations, which did not show any growth after incubation during MIC assays, to $150 \mu \mathrm{L}$ of adequate broth. These preparations were incubated at $37{ }^{\circ} \mathrm{C}$ for $48 \mathrm{~h}$. The $\mathrm{MBC}$ was regarded as the lowest concentration of extract, which did not produce a color change after addition of INT as mentioned above [22, 23].

To evaluate the role of efflux pumps in the susceptibility of Gram-negative bacteria to Xanthosoma mafaffa, Moringa oleifera and Passiflora edulis, crude extracts were tested in the presence of PAßN (at $30 \mu \mathrm{g} / \mathrm{mL}$ ) against ten selected MDR phenotypes (E. coli AG100, AG102 and AG100ATet, E. aerogenes EA289, EA27 and CM64, K. pneumoniae KP55 and KP63, P. aeruginosa PA124 and P. stuartii NAE16).

Extracts from Xanthosoma mafaffa, Moringa oleifera and Passiflora edulis were also tested in association with

Table $4 \mathrm{MIC}$ (FIC) of different antibiotics in association with the extract of Xanthosoma mafaffa at MIC/2, MIC/4 against ten MDR bacteria strains

\begin{tabular}{|c|c|c|c|c|c|c|c|c|c|c|c|c|}
\hline \multirow[t]{2}{*}{ Antibiotics } & \multicolumn{12}{|c|}{ Bacterial strains, MIC $(\mu \mathrm{g} / \mathrm{mL})$ of antibiotics in the absence and presence of the extract } \\
\hline & $\begin{array}{l}\text { Extract } \\
\text { concentration }\end{array}$ & PA124 & AG100 & AG102 & AG100Atet & EA27 & EA289 & CM64 & KP55 & KP63 & NEA16 & $\begin{array}{l}\text { PBSS } \\
\text { (\%) }\end{array}$ \\
\hline \multirow[t]{3}{*}{ CIP } & 0 & 16 & 0.50 & 0.50 & - & 1 & 1 & 0.50 & 2 & - & 1 & \\
\hline & $\mathrm{CMI} / 2$ & $16(1)^{1}$ & $0.50(1)^{1}$ & $<0.50(\mathrm{na})^{\mathrm{S}}$ & $-(\mathrm{na})$ & $0.25(0.25)^{\mathrm{S}}$ & $0.50(0.50)^{\mathrm{s}}$ & $1(2)^{\prime}$ & $<0.50(\mathrm{na})^{\mathrm{S}}$ & $16(n a)^{5}$ & $0.50(0.50)^{\mathrm{s}}$ & 60 \\
\hline & $\mathrm{CMl} / 4$ & $16(1)^{\prime}$ & $0.50(1)^{\prime}$ & $<0.50(\mathrm{na})^{\mathrm{S}}$ & $-(n a)$ & $0.25(0.25)^{S}$ & $1(1)^{\prime}$ & $1(2)^{\prime}$ & $<0.50(\mathrm{na})^{\mathrm{S}}$ & $16(n a)^{5}$ & $1(1)^{\prime}$ & 40 \\
\hline \multirow[t]{3}{*}{ NOR } & 0 & 128 & 2 & 1 & - & 4 & 8 & 4 & 16 & - & 4 & \\
\hline & $\mathrm{CMl} / 2$ & $128(1)^{\prime}$ & $2(1)^{\prime}$ & $<1(n a)^{s}$ & $128(\mathrm{na})^{5}$ & $8(2)^{1}$ & $16(2)^{\prime}$ & $32(8)^{A}$ & $16(1)^{\prime}$ & $128(\mathrm{na})^{5}$ & $8(2)^{\prime}$ & 30 \\
\hline & $\mathrm{CMI} / 4$ & $128(1)^{\prime}$ & $2(1)^{\prime}$ & $<1(n a)^{5}$ & $128(n a)^{s}$ & $32(8)^{\mathrm{A}}$ & $16(2)^{\prime}$ & $32(8)^{A}$ & $16(1)^{\prime}$ & $-(n a)$ & $8(2)^{\prime}$ & 20 \\
\hline \multirow[t]{3}{*}{$\mathrm{CHL}$} & 0 & 64 & 4 & 8 & 64 & 64 & 64 & 256 & 64 & - & 32 & \\
\hline & $\mathrm{CMl} / 2$ & $64(1)^{1}$ & $4(1)^{\prime}$ & $1(0.13)^{S}$ & $32(0.50)^{\mathrm{S}}$ & $4(0.06)^{S}$ & $32(0.50)^{S}$ & $256(1)^{\prime}$ & $16(0.25)^{\mathrm{S}}$ & $-(n a)$ & $16(0.50)^{\mathrm{S}}$ & 60 \\
\hline & $\mathrm{CMI} / 4$ & $64(1)^{\prime}$ & $4(1)^{\prime}$ & $2(0.25)^{S}$ & $64(1)^{\prime}$ & $16(0.25)^{S}$ & $32(0.50)^{S}$ & $256(1)^{\prime}$ & $16(0.25)^{S}$ & $-(n a)$ & $16(0.50)^{S}$ & 50 \\
\hline \multirow[t]{3}{*}{ ERY } & 0 & 128 & 0.50 & 0.50 & - & 16 & 16 & 256 & 32 & - & 32 & \\
\hline & $\mathrm{CMI} / 2$ & $64(0.50)^{5}$ & $<0.50(\mathrm{na})^{\mathrm{s}}$ & $<0.50(\mathrm{na})^{\mathrm{s}}$ & $128(n a)^{S}$ & $8(0.50)^{5}$ & $8(0.50)^{5}$ & $256(1)^{l}$ & $32(1)^{\prime}$ & $128(n a)^{S}$ & $16(0.50)^{\mathrm{s}}$ & 80 \\
\hline & $\mathrm{CMl} / 4$ & $64(0.50)^{\mathrm{S}}$ & $<0.50(\mathrm{na})^{\mathrm{S}}$ & $<0.50(\mathrm{na})^{\mathrm{S}}$ & $128(\mathrm{na})^{\mathrm{S}}$ & $8(0.50)^{5}$ & $8(0.50)^{5}$ & $256(1)^{\prime}$ & $64(2)^{\prime}$ & $128(n a)^{S}$ & $16(0.50)^{\mathrm{s}}$ & 80 \\
\hline \multirow[t]{3}{*}{ KAN } & 0 & 64 & 0.50 & 0.50 & 2 & 8 & 32 & 4 & 16 & - & 16 & \\
\hline & $\mathrm{CMI} / 2$ & $32(0.50)^{5}$ & $<0.50(\mathrm{na})^{\mathrm{s}}$ & $<0.50(\mathrm{na})^{\mathrm{s}}$ & $<0.50(\mathrm{na})^{\mathrm{s}}$ & $4(0.50)^{5}$ & $8(0.25)^{5}$ & $2(0.50)^{5}$ & $16(1)^{\prime}$ & $-(\mathrm{na})$ & $16(1)^{\prime}$ & 70 \\
\hline & $\mathrm{CMI} / 4$ & $64(1)^{\prime}$ & $<0.50(\mathrm{na})^{\mathrm{s}}$ & $1(2)^{\prime}$ & $1(0.50)^{\mathrm{s}}$ & $8(1)^{\prime}$ & $8(0.25)^{s}$ & $4(1)^{\prime}$ & $16(1)^{\prime}$ & $-(n a)$ & $16(1)^{\prime}$ & 30 \\
\hline \multirow[t]{3}{*}{ TET } & 0 & 16 & 32 & 2 & 64 & 32 & 32 & 8 & 2 & 8 & 32 & \\
\hline & $\mathrm{CMI} / 2$ & $8(0.50)^{5}$ & $16(0.50)^{\mathrm{S}}$ & $<0.50(\mathrm{na})^{\mathrm{s}}$ & $4(0.06)^{5}$ & $8(0.25)^{5}$ & $4(0.13)^{5}$ & $2(0.25)^{5}$ & $<0.50(\mathrm{na})^{\mathrm{s}}$ & $1(0.13)^{\mathrm{S}}$ & $4(0.13)^{5}$ & 100 \\
\hline & $\mathrm{CMI} / 4$ & $16(1)^{\prime}$ & $32(1)^{\prime}$ & $1(0.50)^{S}$ & $4(0.06)^{5}$ & $8(0.25)^{S}$ & $16(0.50)^{\mathrm{s}}$ & $2(0.25)^{\mathrm{S}}$ & $<0.50(\mathrm{na})^{\mathrm{S}}$ & $1(0.13)^{S}$ & $4(0.13)^{5}$ & 80 \\
\hline \multirow[t]{3}{*}{ AMP } & 0 & - & - & 128 & - & - & - & - & - & - & - & \\
\hline & $\mathrm{CMI} / 2$ & $-(n a)$ & $-(n a)$ & $128(1)^{\prime}$ & $-(n a)$ & -(na) & $-(\mathrm{na})$ & -(na) & $128(\mathrm{na})^{\mathrm{S}}$ & $-(n a)$ & -(na) & 10 \\
\hline & $\mathrm{CMl} / 4$ & $-(n a)$ & $-(n a)$ & $128(1)^{\prime}$ & $-(\mathrm{na})$ & $-(\mathrm{na})$ & $-(\mathrm{na})$ & -(na) & -(na) & $-(n a)$ & -(na) & 00 \\
\hline \multirow[t]{3}{*}{ CEF } & 0 & - & 64 & 32 & - & - & - & - & - & - & - & \\
\hline & $\mathrm{CMI} / 2$ & $-(n a)$ & $64(1)^{\prime}$ & $32(1)^{\prime}$ & $-(\mathrm{na})$ & -(na) & $-(n a)$ & $-(n a)$ & $256(\mathrm{na})^{\mathrm{S}}$ & $-(n a)$ & -(na) & 10 \\
\hline & $\mathrm{CMI} / 4$ & -(na) & $64(1)^{\prime}$ & $32(1)^{\prime}$ & -(na) & -(na) & -(na) & $-(\mathrm{na})$ & -(na) & -(na) & -(na) & 00 \\
\hline
\end{tabular}

${ }^{a}$ Antibiotics [TET tetracycline, CIP ciprofloxacin, NOR norfloxacin, KAN kanamycin, CHL chloramphenicol, ERY erythromycin, AMP ampicillin, CEF cefepime] ${ }^{\mathrm{b}}$ Bacterial strains: Escherichia coli [AG100, AG102, AG100Atet], Pseudomonas aeruginosa [PA124], Enterobacter aerogenes [CM64, EA27, EA289], Klebsiella pneumonia [KP55], Providencia stuartii [NAE16]

'PBSS: percentage of bacteria strain on which synergism has been observed; (): FIC (Fractional Inhibitory Concentration) of the antibiotics after association with plants extract; S: Synergy, I: Indifference; na: not applicable; The values in bold represent the cases of synergy between extract and antibiotic; $(-):>256 \mu \mathrm{g} / \mathrm{mL}$ 
antibiotics at their sub-inhibitory concentrations as obtained in each bacterium (MIC/2 and $\mathrm{MIC} / 4)[4,5,11]$ against ten MDR phenotypes. Fractional inhibitory concentration (FIC) was calculated as the ratio of $\mathrm{MIC}_{\text {Antibiotic in combination }} / \mathrm{MIC}_{\text {Antibiotic alone }}$ and the results were discussed as follows: synergy $(\leq 0.5)$, indifferent $(>0.5$ to 4$)$, or antagonism $(>4)[26,27]$. All assays were performed in triplicate.

\section{Results}

\section{Phytochemical composition}

The results of the phytochemical screening (Table 2) showed that all the tested plant extracts contain polyphenols, triterpenes, sterols and saponins. The other classes of secondary metabolites were selectively distributed. Also, the extract from $M$. oleifera contains all the classes of screened secondary metabolites.

\section{Antibacterial activity}

Results of the antibacterial activities of the tested extracts alone and in some cases in the presence of the $\mathrm{PA} \beta \mathrm{N}$ on a panel of 19 Gram-negative bacteria are summarized in Table 3. It appears that the extracts from $P$. edulis inhibited the growth of $17 / 19$ (89.5 \%) bacteria with a concentration ranged from 128 to $1024 \mu \mathrm{g} / \mathrm{mL}$. The two other samples showed selective activities, their inhibitory activity being recorded on 13/19 (68.4\%) and 11/19 (57.9\%) tested bacteria for $M$. oleifera and X. mafaffa extracts respectively. The lowest MIC value $(128 \mu \mathrm{g} / \mathrm{mL})$ was

Table 5 MIC (FIC) of different antibiotics in association of the extract of Passiflora edulis at MIC/2, MIC/4 against ten MDR bacteria strains

\begin{tabular}{|c|c|c|c|c|c|c|c|c|c|c|c|c|}
\hline \multirow[t]{2}{*}{ Antibiotics } & \multicolumn{12}{|c|}{ Bacterial strains, MIC $(\mu \mathrm{g} / \mathrm{mL})$ of antibiotics in the absence and presence of the extract } \\
\hline & $\begin{array}{l}\text { Extract } \\
\text { concentration }\end{array}$ & PA124 & AG100 & AG102 & AG100Atet & EA27 & EA289 & CM64 & KP55 & KP63 & NEA16 & $\begin{array}{l}\text { PBSS } \\
(\%)\end{array}$ \\
\hline \multirow[t]{3}{*}{ CIP } & 0 & 16 & 0.50 & 0.50 & - & 1 & 1 & 0.50 & 2 & - & 1 & \\
\hline & $\mathrm{CMl} / 2$ & $16(1)^{\prime}$ & $0.50(1)^{\prime}$ & $0.50(1)^{\prime}$ & $-(n a)$ & $2(2)^{\prime}$ & $<0.50(\mathrm{na})^{\mathrm{S}}$ & $8(16)^{A}$ & $<0.50(\mathrm{na})^{\mathrm{S}}$ & $-(n a)$ & $1(1)^{1}$ & 20 \\
\hline & $\mathrm{CMl} / 4$ & $16(1)^{\prime}$ & $0.50(1)^{\prime}$ & $0.50(1)^{\prime}$ & $-(n a)$ & $4(4)^{A}$ & $1(1)^{\prime}$ & $64(256)^{A}$ & $<0.50(\mathrm{na})^{\mathrm{S}}$ & $-(n a)$ & $1(1)^{\prime}$ & 10 \\
\hline \multirow[t]{3}{*}{ NOR } & 0 & 128 & 2 & 1 & - & 4 & 8 & 4 & 16 & - & 4 & \\
\hline & $\mathrm{CMl} / 2$ & $128(1)^{\prime}$ & $2(1)^{\prime}$ & $<1(n a)^{5}$ & $128(n a)^{s}$ & $4(1)^{\prime}$ & $4(0.50)^{S}$ & $64(16)^{A}$ & $4(0.25)^{5}$ & -(na) & $4(1)^{1}$ & 40 \\
\hline & $\mathrm{CMl} / 4$ & $128(1)^{\prime}$ & $2(1)^{\prime}$ & $<1(n a)^{5}$ & $128(\mathrm{na})^{\mathrm{S}}$ & $16(4)^{\prime}$ & $8(1)^{\prime}$ & $-(\mathrm{na})^{\mathrm{A}}$ & $4(0.25)^{5}$ & -(na) & $4(1)^{1}$ & 30 \\
\hline \multirow[t]{3}{*}{$\mathrm{CHL}$} & 0 & 64 & 4 & 8 & 64 & 64 & 64 & 256 & 64 & - & 32 & \\
\hline & $\mathrm{CMI} / 2$ & $64(1)^{\prime}$ & $2(0.50)^{\mathrm{S}}$ & $<0.50(\mathrm{na})^{\mathrm{s}}$ & $32(0.50)^{\mathrm{S}}$ & $2(0.03)^{\mathrm{S}}$ & $32(0.50)^{\mathrm{s}}$ & $128(0.50)^{\mathrm{S}}$ & $64(1)^{\prime}$ & $-(\mathrm{na})$ & $16(0.50)^{\mathrm{S}}$ & 70 \\
\hline & $\mathrm{CMl} / 4$ & $64(1)^{\prime}$ & $2(0.50)^{\mathrm{S}}$ & $1(0.13)^{S}$ & $32(0.50)^{\mathrm{S}}$ & $16(0.25)^{\mathrm{S}}$ & $64(1)^{\prime}$ & $256(1)^{1}$ & $64(1)^{\prime}$ & -(na) & $16(0.50)^{\mathrm{s}}$ & 50 \\
\hline \multirow[t]{3}{*}{ ERY } & 0 & 128 & 0.50 & 0.50 & - & 16 & 16 & 256 & 32 & - & 32 & \\
\hline & $\mathrm{CMI} / 2$ & $64(0.50)^{5}$ & $<0.50(\mathrm{na})^{\mathrm{s}}$ & $<0.50(\mathrm{na})^{\mathrm{s}}$ & $-(n a)$ & $128(8)^{A}$ & $1(0.06)^{5}$ & $256(1)^{\prime}$ & $8(0.25)^{5}$ & $128(\mathrm{na})^{\mathrm{S}}$ & $16(0.50)^{5}$ & 70 \\
\hline & $\mathrm{CMl} / 4$ & $128(1)^{\prime}$ & $<0.50(\mathrm{na})^{\mathrm{S}}$ & $<0.50(\mathrm{na})^{\mathrm{s}}$ & $-(n a)$ & $128(8)^{\mathrm{A}}$ & $16(1)^{\prime}$ & $256(1)^{\prime}$ & $2(0.06)^{S}$ & $128(\mathrm{na})^{\mathrm{S}}$ & $16(0.50)^{S}$ & 50 \\
\hline \multirow[t]{3}{*}{ KAN } & 0 & 64 & 0.50 & 0.50 & 2 & 8 & 32 & 4 & 16 & - & 16 & \\
\hline & $\mathrm{CMl} / 2$ & $64(1)^{\prime}$ & $<0.50(\mathrm{na})^{\mathrm{S}}$ & $0.50(1)^{\prime}$ & $<0.50(\mathrm{na})^{\mathrm{S}}$ & $4(0.50)^{S}$ & $2(0.06)^{S}$ & $128(32)^{\mathrm{A}}$ & $2(0.13)^{\mathrm{S}}$ & $-(n a)$ & $16(1)^{\prime}$ & 60 \\
\hline & $\mathrm{CMl} / 4$ & $64(1)^{\prime}$ & $<0.50(\mathrm{na})^{\mathrm{S}}$ & $0.50(1)^{\prime}$ & $1(0.50)^{S}$ & $4(0.50)^{S}$ & $8(0.25)^{S}$ & -(na) & $2(0.13)^{S}$ & -(na) & $16(1)^{\prime}$ & 60 \\
\hline \multirow[t]{3}{*}{ TET } & 0 & 16 & 32 & 2 & 64 & 32 & 32 & 8 & 2 & 8 & 32 & \\
\hline & $\mathrm{CMI} / 2$ & $8(0.50)^{5}$ & $16(0.50)^{s}$ & $<0.50(\mathrm{na})^{\mathrm{s}}$ & $8(0.13)^{5}$ & $64(2)^{\prime}$ & $2(0.06)^{S}$ & $32(4)^{\mathrm{A}}$ & $<0.50(n a)^{S}$ & $4(0.50)^{\mathrm{S}}$ & $8(0.25)^{5}$ & 80 \\
\hline & $\mathrm{CMI} / 4$ & $16(1)^{\prime}$ & $16(0.50)^{\mathrm{s}}$ & $<0.50(\mathrm{na})^{\mathrm{s}}$ & $8(0.13)^{5}$ & $64(2)^{\prime}$ & $4(0.13)^{5}$ & $64(8)^{A}$ & $<0.50(\mathrm{na})^{\mathrm{S}}$ & $4(0.50)^{5}$ & $8(0.25)^{5}$ & 70 \\
\hline \multirow[t]{3}{*}{ AMP } & 0 & - & - & 128 & - & - & - & - & - & - & - & \\
\hline & $\mathrm{CMI} / 2$ & $-(n a)$ & -(na) & $<1(n a)^{5}$ & $-(n a)$ & $-(n a)$ & $-(n a)$ & $-(n a)$ & $-(n a)$ & $256(\mathrm{na})^{\mathrm{S}}$ & $-(n a)$ & 20 \\
\hline & $\mathrm{CMI} / 4$ & $-(n a)$ & $-(n a)$ & $32(0.25)^{S}$ & $-(n a)$ & $-(n a)$ & $-(n a)$ & -(na) & $-(n a)$ & $256(\mathrm{na})^{S}$ & $-(n a)$ & 20 \\
\hline \multirow[t]{3}{*}{ CEF } & 0 & - & 64 & 32 & - & - & - & - & - & - & - & \\
\hline & $\mathrm{CMl} / 2$ & $-(n a)$ & $128(2)^{\prime}$ & $8(0.25)^{S}$ & $-(n a)$ & $-(n a)$ & $-(n a)$ & $-(n a)$ & -(na) & $128(n a)^{S}$ & $-(n a)$ & 20 \\
\hline & $\mathrm{CMl} / 4$ & $-(n a)$ & $128(2)^{\prime}$ & $32(1)^{\prime}$ & -(na) & $-(n a)$ & $-(n a)$ & $-(n a)$ & $-(n a)$ & $128(n a)^{S}$ & $-(n a)$ & 10 \\
\hline
\end{tabular}

${ }^{a}$ Antibiotics [TET tetracycline, CIP ciprofloxacin, NOR norfloxacin, KAN kanamycin, CHL chloramphenicol, ERY erythromycin, AMP ampicillin, CEF cefepime] ${ }^{\mathrm{b}}$ Bacterial strains: Escherichia coli [AG100, AG102, AG100Atet], Pseudomonas aeruginosa [PA124], Enterobacter aerogenes [CM64, EA27, EA289], Klebsiella pneumonia [KP55], Providencia stuartii [NAE16].

'PBSS: percentage of bacteria strain on which synergism has been observed; (): FIC (Fractional Inhibitory Concentration) of the antibiotics after association with plants extract; S: Synergy, I: Indifference; na: not applicable; The values in bold represent the cases of synergy between extract and antibiotic; $(-):>256 \mu \mathrm{g} / \mathrm{mL}$ 
obtained with $P$. edulis and M. oleifera extracts on Escherichia coli AG100.

\section{Role of efflux pumps in the susceptibility of Gram-negative bacteria}

Ten of the studied MDR bacteria were also tested for their susceptibility to the plant extracts in the presence of the PA $\beta N$ at $30 \mu \mathrm{g} / \mathrm{mL}$. The results showed that when combined with the extracts, PA $\beta \mathrm{N}$ improves the activity (decrease of MIC values) of X. mafaffa on 4/10 (40\%) of tested MDR strains. The EPI also improved the activity of $P$. edulis against $E$. coli AG100 (Table 3).

Effects of the association of the extracts with antibiotics A preleminary study was performed against $P$. aeruginosa PA124. This allowed selection of the appropriate sub-inhibitory concentrations of $\mathrm{MIC} / 2$ and $\mathrm{MIC} / 4$ for further studies. All the three extracts were combined separately with eight antibiotics (CIP, NOR, CHL, ERY, KAN, TET, CEF and AMP) to evaluate their possible synergetic effects. The results summarized in Tables 4, 5 and 6 showed synergic effects of the three tested extract with most of tested antibiotics except $\beta$-lactams (CEF and AMP). At MIC/2 of the extract from $X$. mafaffa, synergistic effects were observed with 6/8 (75 \%) antibiotics (CIP, NOR, CHL, ERY, KAN, TET) against the tested MDR bacteria. Synergistic effects (FIC ranging from 0.5 to 0.03 ) were noted with the associations of each of the $X$. mafaffa, $P$. edulis and M. oleifera extracts and antibiotics. Low FIC values of 0.03 were obtained with the associations of $M$. oleifera extracts + ERY and M. oleifera extract + CHL against Enterobacter aerogenes EA27.

Table $6 \mathrm{MIC}$ (FIC) of different antibiotics after the association of the extract of Moringa oleifera at MIC/2, MIC/4 against ten MDR bacteria strains

\begin{tabular}{|c|c|c|c|c|c|c|c|c|c|c|c|c|}
\hline \multirow[t]{2}{*}{ Antibiotics } & \multicolumn{12}{|c|}{ Bacterial strains, MIC $(\mu \mathrm{g} / \mathrm{mL})$ of antibiotics in the absence and presence of the extract } \\
\hline & $\begin{array}{l}\text { Extract } \\
\text { concentration }\end{array}$ & PA124 & AG100 & AG102 & AG100Atet & EA27 & EA289 & CM64 & KP55 & KP63 & NEA16 & $\begin{array}{l}\text { PBSS } \\
(\%)\end{array}$ \\
\hline \multirow[t]{3}{*}{ CIP } & 0 & 16 & 0.50 & 0.50 & - & 1 & 1 & 0.50 & 2 & - & 1 & \\
\hline & $\mathrm{CMl} / 2$ & $32(2)^{1}$ & $0.50(1)^{1}$ & $0.50(1)^{1}$ & $-(\mathrm{na})$ & $1(1)^{1}$ & $0.50(0.50)^{\mathrm{s}}$ & $4(8)^{\mathrm{A}}$ & $<0.50(\mathrm{na})^{\mathrm{S}}$ & $-(\mathrm{na})$ & $1(1)^{1}$ & 20 \\
\hline & $\mathrm{CMI} / 4$ & $32(2)^{\prime}$ & $0.50(1)^{\prime}$ & $0.50(1)^{\prime}$ & $-(n a)$ & $1(1)^{1}$ & $0.50(0.50)^{\mathrm{s}}$ & $8(16)^{A}$ & $<0.50(\mathrm{na})^{\mathrm{S}}$ & $-(n a)$ & $1(1)^{\prime}$ & 20 \\
\hline \multirow[t]{3}{*}{ NOR } & 0 & 128 & 2 & 1 & - & 4 & 8 & 4 & 16 & - & 4 & \\
\hline & $\mathrm{CMl} / 2$ & $128(1)^{\prime}$ & $2(1)^{\prime}$ & $<1(n a)^{5}$ & $-(\mathrm{na})$ & $4(1)^{1}$ & $4(0.50)^{5}$ & $64(16)^{A}$ & $8(0.50)^{5}$ & -(na) & $2(0.50)^{5}$ & 40 \\
\hline & $\mathrm{CMl} / 4$ & $128(1)^{\prime}$ & $2(1)^{\prime}$ & $<1(n a)^{5}$ & $-(n a)$ & $4(1)^{1}$ & $4(0.50)^{S}$ & $128(32)^{\mathrm{A}}$ & $16(1)^{\prime}$ & $-(n a)$ & $2(0.50)^{S}$ & 30 \\
\hline \multirow[t]{3}{*}{$\mathrm{CHL}$} & 0 & 64 & 4 & 8 & 64 & 64 & 64 & 256 & 64 & - & 32 & \\
\hline & $\mathrm{CMl} / 2$ & $32(0.50)^{\mathrm{S}}$ & $4(1)^{\prime}$ & $1(0.13)^{\mathrm{S}}$ & $64(1)^{\prime}$ & $32(0.50)^{\mathrm{S}}$ & $32(0.50)^{5}$ & $128(0.50)^{\mathrm{S}}$ & $2(0.03)^{S}$ & -(na) & $32(1)^{\prime}$ & 60 \\
\hline & $\mathrm{CMl} / 4$ & $64(1)^{\prime}$ & $4(1)^{1}$ & $2(0.25)^{S}$ & $64(1)^{\prime}$ & $32(0.50)^{\mathrm{S}}$ & $32(0.50)^{\mathrm{S}}$ & $256(1)^{\prime}$ & $4(0.06)^{5}$ & $-(\mathrm{na})$ & $32(1)^{1}$ & 40 \\
\hline \multirow[t]{3}{*}{ ERY } & 0 & 128 & 0.50 & 0.50 & - & 16 & 16 & 256 & 32 & - & 32 & \\
\hline & $\mathrm{CMI} / 2$ & $128(1)^{\prime}$ & $<0.50(\mathrm{na})^{\mathrm{s}}$ & $<0.50(\mathrm{na})^{\mathrm{s}}$ & $128(n a)^{S}$ & $8(0.50)^{5}$ & $8(0.50)^{5}$ & $128(0.50)^{5}$ & $1(0.03)^{\mathrm{S}}$ & $128(n a)^{s}$ & $16(0.50)^{\mathrm{s}}$ & 90 \\
\hline & $\mathrm{CMl} / 4$ & $128(1)^{\prime}$ & $<0.50(\mathrm{na})^{\mathrm{S}}$ & $<0.50(\mathrm{na})^{\mathrm{S}}$ & $128(\mathrm{na})^{\mathrm{S}}$ & $8(0.50)^{5}$ & $8(0.50)^{5}$ & $256(1)^{\prime}$ & $4(0.13)^{5}$ & $128(n a)^{S}$ & $16(0.50)^{\mathrm{s}}$ & 80 \\
\hline \multirow[t]{3}{*}{ KAN } & 0 & 64 & 0.50 & 0.50 & 2 & 8 & 32 & 4 & 16 & - & 16 & \\
\hline & $\mathrm{CMI} / 2$ & $32(0.50)^{5}$ & $<0.50(\mathrm{na})^{\mathrm{S}}$ & $<0.50(\mathrm{na})^{\mathrm{s}}$ & $<0.50(\mathrm{na})^{\mathrm{s}}$ & $1(0.13)^{5}$ & $2(0.06)^{S}$ & $8(2)^{\prime}$ & $4(0.25)^{S}$ & -(na) & $4(0.25)^{5}$ & 80 \\
\hline & $\mathrm{CMI} / 4$ & $64(1)^{\prime}$ & $<0.50(n a)^{s}$ & $<0.50(n a)^{s}$ & $1(0.50)^{5}$ & $2(0.25)^{5}$ & $4(0.13)^{5}$ & $256(64)^{A}$ & $8(0.50)^{5}$ & -(na) & $8(0.50)^{5}$ & 70 \\
\hline \multirow[t]{3}{*}{ TET } & 0 & 16 & 32 & 2 & 64 & 32 & 32 & 8 & 2 & 8 & 32 & \\
\hline & $\mathrm{CMI} / 2$ & $16(1)^{\prime}$ & $16(0.50)^{\mathrm{S}}$ & $1(0.50)^{S}$ & $8(0.13)^{S}$ & $16(0.50)^{\mathrm{S}}$ & $4(0.13)^{5}$ & $8(1)^{\prime}$ & $<0.50(\mathrm{na})^{\mathrm{S}}$ & $4(0.50)^{5}$ & $8(0.25)^{5}$ & 80 \\
\hline & $\mathrm{CMI} / 4$ & $16(1)^{\prime}$ & $32(1)^{1}$ & $1(0.50)^{S}$ & $8(0.13)^{5}$ & $16(0.50)^{\mathrm{S}}$ & $4(0.13)^{5}$ & $64(8)^{\mathrm{A}}$ & $<0.50(\mathrm{na})^{\mathrm{s}}$ & $4(0.50)^{5}$ & $8(0.25)^{5}$ & 70 \\
\hline \multirow[t]{3}{*}{ AMP } & 0 & - & - & 128 & - & - & - & - & - & - & - & \\
\hline & $\mathrm{CMl} / 2$ & -(na) & $-(n a)$ & $128(1)^{\prime}$ & -(na) & $-(n a)$ & $-(n a)$ & -(na) & -(na) & -(na) & -(na) & 00 \\
\hline & $\mathrm{CMl} / 4$ & $-(\mathrm{na})$ & $-(n a)$ & $128(1)^{\prime}$ & $-(n a)$ & $-(\mathrm{na})$ & $-(\mathrm{na})$ & -(na) & -(na) & -(na) & -(na) & 00 \\
\hline \multirow[t]{3}{*}{ CEF } & 0 & - & 64 & 32 & - & - & - & - & - & - & - & \\
\hline & $\mathrm{CMI} / 2$ & $-(\mathrm{na})$ & $64(1)^{1}$ & $32(1)^{\prime}$ & $-(n a)$ & $-(n a)$ & $-(\mathrm{na})$ & $-(n a)$ & $-(n a)$ & $-(n a)$ & $-(n a)$ & 00 \\
\hline & $\mathrm{CMI} / 4$ & $-(\mathrm{na})$ & $64(1)^{\prime}$ & $32(1)^{\prime}$ & -(na) & -(na) & -(na) & $-(\mathrm{na})$ & -(na) & $-(\mathrm{na})$ & -(na) & 00 \\
\hline
\end{tabular}

antibiotics [TET tetracycline, CIP ciprofloxacin, NOR norfloxacin, KAN kanamycin, CHL chloramphenicol, ERY erythromycin, AMP ampicillin, CEF: cefepime] bBacterial strains: Escherichia coli [AG100, AG102, AG100Atet], Pseudomonas aeruginosa [PA124], Enterobacter aerogenes [CM64, EA27, EA289], Klebsiella pneumonia [KP55], Providencia stuartii [NAE16]

CPBSS: percentage of bacteria strain on which synergism has been observed; (): FIC (Fractional Inhibitory Concentration) of the antibiotics after association with plants extract; S: Synergy, I: Indifference; na: not applicable; The values in bold represent the cases of synergy between extract and antibiotic; $(-):>256 \mu \mathrm{g} / \mathrm{mL}$ 


\section{Discussion}

Phytochemical screening revealed the presence of several classes of secondary metabolites such as alkaloids, polyphenols, flavonoids, anthraquinones, coumarins, saponins, tannins, triterpenes and steroids. Several molecules belonging to these classes were found to be active on pathogenic microorganisms [3, 28-30]. The presence of such metabolites in the studied plant extracts can provide a preliminary explanation on their antibacterial activities. Differences were observed in the antibacterial activities of the extracts. These could be due to the differences in their chemical composition as well as in the mechanism of action of their bioactive constituents [3]. As shown in Table 2, all the extracts are rich in secondary metabolites especially the extract from M. oleifera (which contains all the tested classes); However, activity does not depend only on the presence of secondary metabolites in the plant extracts, but mostly on their concentration and the possible interactions with other constituents.

To the best of our knowledge, the antibacterial activity of $X$. mafaffa is being reported here for the first time. The inhibitory activity of $M$. oleifera was previously reported against some bacteria such as Escherichia coli, Pseudomaonas aeruginosa, Stapylococcus aureus and Salmonella typhii [31]. The present study confirmed the antimicrobial potential of this plant and provide additional information on it ability to inhibit the growth of MDR bacteria.

The results of this work are very important taking in account the medicinal importance of the tested MDR bacteria [32-36] and also the fact that samples used are edible plants. In the presence of PA $\beta N$ (EPI), the antibacterial activity of some of the extracts increased, suggesting that some active constituents may have intracellular target. In the presence of the EPI, the activity of $M$. oleifera remain unchanged, indicating that the bioactive compounds of this extract are not the substrates of bacterial efflux pumps, as the tested MDR bacteria over-express efflux pumps [32-36]. However, it should be observed that in certain cases (Table 3), MIC values of Xanthosoma mafaffa and Passiflora edulis extracts increased in the presence of PAßN. A possible explanation is that some active constituents of these extracts may act in the cell coat, inhibiting the synthesis of peptidoglycan. In such case, in the absence of PAßN, such compounds are extruded from the cytoplasm of bacteria by efflux pumps, then re-cross the cell membrane to reach their target in the coat, explaining while the MIC value is lower; in presence of PAßN, the efflux pumps are blocked and such compounds could not be expelled from the cytoplasm, reducing their concentration in the cell coat and consequently their activity, explaining their higher MIC values.
In the recent years, scientists intensified the search of substances with ability to restore the activity of available antibiotics to MDR bacteria. In this work, synergistic effects were noted with the associations of $X$. mafaffa, $P$. edulis and $M$. oleifera extracts and some antibiotics, providing additional information of their possible use to combat MDR phenotypes.

\section{Conclusion}

The results of the present investigation show that $X$. mafaffa, $P$. edulis and $M$. oleifera may be useful in the control of many infectious diseases, particularly those caused by the multidrug resistant Gram-negative bacteria. These extracts may be used alone or in combination with certain antibiotics such as tetracycline, ciprofloxacin, norfloxacin, chloramphenicol, erythromycin, kanamycin but not beta-lactamines. The isolation of the active compounds from the three plants constitutes the limitation of the present study and will be further performed. Also, further investigations of the plant extracts are warranted in vivo to validate their use for the control of infectious diseases.

\section{Abbreviations \\ AMP: Ampicillin; ATCC: American type culture collection; CEF: Cefepime; CFU: Colony forming unit; CHL: Chloramphenicol; CIP: Ciprofloxacin; DMSO: Dimethylsulfoxyde; EPI: Efflux pump inhibitor; ERY: Erythromycin; FIC: Fractional inhibitory concentration; INT: p-iodonitrotetrazolium chloride; KAN: Kanamycin; MDR: Multidrug resistant; MHB: Mueller hinton broth; MIC: Minimal inhibitory concentration; NOR: Norfloxacin; PAßN: Phenylalanine arginine-ß-Naphthylamide; RND: Resistance nodulation-cell division; \\ STR: Streptomycin; TET: Tetracycline.}

Competing interests

The authors declare that there are no conflict of interests.

Authors' contributions

JKD and FTK carried out the study; VK supervised the work, designed the experiments, wrote the manuscript, provided the bacterial strains and chemicals; all authors read and approved the final manuscript.

\section{Acknowledgements}

Authors are thankful to the Cameroon National Herbarium for identification of plants.

Received: 16 June 2015 Accepted: 8 January 2016

Published online: 11 January 2016

References

1. WHO: World Health Statistics 2013. http://www.whoint/gho/publications/ world_health_statistics/2013/en/2013, Accessed on February 2014.

2. Poole K. Mechanisms of bacterial biocide and antibiotic resistance. J Appl Microbiol. 2002;92(Suppl):55S-64.

3. Cowan MM. Plant products as antimicrobial agents. Clin Microbiol Rev. 1999:12:564-82.

4. Fankam AG, Kuete V, Voukeng IK, Kuiate JR, Pages JM. Antibacterial activities of selected Cameroonian spices and their synergistic effects with antibiotics against multidrug-resistant phenotypes. BMC Complement Altern Med. 2011;11:104

5. Voukeng IK, Kuete V, Dzoyem JP, Fankam AG, Noumedem JA, Kuiate JR, et al. Antibacterial and antibiotic-potentiation activities of the methanol extract of some cameroonian spices against Gram-negative multi-drug resistant phenotypes. BMC Res Notes. 2012;5:299. 
6. Djeussi DE, Noumedem JA, Seukep JA, Fankam AG, Voukeng IK, Tankeo SB, et al. Antibacterial activities of selected edible plants extracts against multidrug-resistant Gram-negative bacteria. BMC Complement Altern Med. 2013;13:164.

7. Seukep JA, Fankam AG, Djeussi DE, Voukeng IK, Tankeo SB, Noumdem JA, et al. Antibacterial activities of the methanol extracts of seven Cameroonian dietary plants against bacteria expressing MDR phenotypes. Springerplus. 2013;2:363.

8. Kuete V, Ngameni B, Tangmouo JG, Bolla JM, Alibert-Franco S, Ngadjui BT, et al. Efflux pumps are involved in the defense of Gram-negative bacteria against the natural products isobavachalcone and diospyrone. Antimicrob Agents Chemother. 2010;54:1749-52.

9. Kuete $\mathrm{V}$, Alibert-Franco S, Eyong KO, Ngameni B, Folefoc GN, Nguemeving JR, et al. Antibacterial activity of some natural products against bacteria expressing a multidrug-resistant phenotype. Int J Antimicrob Agents. 2011;37:156-61.

10. Noumedem JA, Mihasan M, Lacmata ST, Stefan M, Kuiate JR, Kuete V. Antibacterial activities of the methanol extracts of ten Cameroonian vegetables against Gram-negative multidrug-resistant bacteria. BMC Complement Altern Med. 2013;13:26.

11. Noumedem JA, Mihasan M, Kuiate JR, Stefan M, Cojocaru D, Dzoyem JP, et al. In vitro antibacterial and antibiotic-potentiation activities of four edible plants against multidrug-resistant gram-negative species. BMC Complement Altern Med. 2013;13:190

12. Fankam AG, Kuiate JR, Kuete V. Antibacterial activities of Beilschmiedia obscura and six other Cameroonian medicinal plants against multi-drug resistant Gramnegative phenotypes. BMC Complement Altern Med. 2014;14:241.

13. Touani FK, Seukep AJ, Djeussi DE, Fankam AG, Noumedem JA, Kuete V. Antibiotic-potentiation activities of four Cameroonian dietary plants against multidrug-resistant Gram-negative bacteria expressing efflux pumps. BMC Complement Altern Med. 2014;14:258.

14. Harbone J. Phytochemical methods: a guide to modern techniques of plant analysis. London: Chapman \& Hall; 1973

15. Ngameni B, Fotso GW, Kamga J, Ambassa P, Abdou T, Fankam AG, et al. 9 Flavonoids and Related Compounds from the Medicinal Plants of Africa. In: Kuete V, editor. Medicinal Plant Research in Africa. Oxford: Elsevier; 2013. p. 301-50.

16. Wansi JD, Devkota KP, Tshikalange E, Kuete V. 14 - Alkaloids from the Medicinal Plants of Africa. In: Kuete V, editor. Medicinal Plant Research in Africa. Oxford: Elsevier; 2013. p. 557-605.

17. Poumale HMP, Hamm R, Zang Y, Shiono Y, Kuete V. 8 - Coumarins and Related Compounds from the Medicinal Plants of Africa. In: Kuete V, editor. Medicinal Plant Research in Africa. Oxford: Elsevier; 2013. p. 261-300.

18. Eloff JN. A sensitive and quick microplate method to determine the minimal inhibitory concentration of plant extracts for bacteria. Planta Med. 1998;64:711-3.

19. Mativandlela SPN, Lall N, Meyer JJM. Antibacterial, antifungal and antitubercular activity of (the roots of) Pelargonium reniforme (CURT) and Pelargonium sidoides (DC) (Geraniaceae) root extracts. S Afr J Bot. 2006;72:232-7

20. Lacmata ST, Kuete V, Dzoyem JP, Tankeo SB, Teke GN, Kuiate JR, et al. Antibacterial activities of selected Cameroonian plants and their synergistic effects with antibiotics against bacteria expressing MDR phenotypes. Evid Based Complement Altern Med. 2012;2012:623723

21. Kuete V, Kamga J, Sandjo LP, Ngameni B, Poumale HM, Ambassa P, et al Antimicrobial activities of the methanol extract, fractions and compounds from Ficus polita Vahl. (Moraceae). BMC Complement Altern Med. 2011;11:6.

22. Kuete V, Nana F, Ngameni B, Mbaveng AT, Keumedjio F, Ngadjui BT. Antimicrobial activity of the crude extract, fractions and compounds from stem bark of Ficus ovata (Moraceae). J Ethnopharmacol. 2009;124:556-61.

23. Kuete V, Wansi JD, Mbaveng AT, Kana Sop MM, Tadjong AT, Beng VP, et al, Antimicrobial activity of the methanolic extract and compounds from Teclea afzelii (Rutaceae). S Afr J Bot. 2008;74:572-6.

24. Kuete V, Ngameni B, Simo CC, Tankeu RK, Ngadjui BT, Meyer JJ, et al. Antimicrobial activity of the crude extracts and compounds from Ficus chlamydocarpa and Ficus cordata (Moraceae). J Ethnopharmacol. 2008;120:17-24.

25. Kuete $V$, Wabo GF, Ngameni B, Mbaveng AT, Metuno R, Etoa FX, et al. Antimicrobial activity of the methanolic extract, fractions and compounds from the stem bark of Irvingia gabonensis (Ixonanthaceae). J Ethnopharmacol. 2007;114:54-60.

26. Shahverdi AR, Monsef-Esfahani HR, Tavasoli F, Zaheri A, Mirjani R. TransCinnamaldehyde from Cinnamomum zeylanicum Bark Essential Oil Reduces the Clindamycin Resistance of Clostridium difficile in vitro. J Food Sci. 2007;72:S055-8.
27. Braga LC, Leite AAM, Xavier KGS, Takahashi JA, Bemquerer MP, ChartoneSouza $\mathrm{E}$, et al. Synergic interaction between pomegranate extract and antibiotics against Staphylococcus aureus. Can J Microbiol. 2005;51:541-7.

28. Awouafack MD, Tane P, Kuete V, Eloff JN. 2 - Sesquiterpenes from the Medicinal Plants of Africa. In: Kuete V, editor. Medicinal Plant Research in Africa. Oxford: Elsevier; 2013. p. 33-103.

29. Ndhlala AR, Amoo SO, Ncube B, Moyo M, Nair JJ, Van Staden J. 16 - Antibacterial, antifungal, and antiviral activities of African medicinal plants. In: Kuete V, editor. Medicinal Plant Research in Africa. Oxford: Elsevier; 2013. p. 621-59.

30. Tsopmo A, Awah FM, Kuete V. 12 - Lignans and Stilbenes from African Medicinal Plants. In: Kuete V, editor. Medicinal Plant Research in Africa. Oxford: Elsevier; 2013. p. 435-78.

31. Vinoth B, Manivasagaperumal R, Balamurugan S. Phytochemical analysis and antibacterial activity of Moringa oleifera Lam. Int J Res Biol Sci. 2012;2:98-102.

32. Mallea M, Chevalier J, Bornet C, Eyraud A, Davin-Regli A, Bollet C, et al. Porin alteration and active efflux: two in vivo drug resistance strategies used by Enterobacter aerogenes. Microbiology. 1998;144(Pt 11):3003-9.

33. Chevalier J, Pages JM, Eyraud A, Mallea M. Membrane permeability modifications are involved in antibiotic resistance in Klebsiella pneumoniae. Biochem Biophys Res Commun. 2000;274(2):496-9.

34. Pradel E, Pages JM. The AcrAB-TolC efflux pump contributes to multidrug resistance in the nosocomial pathogen Enterobacter aerogenes. Antimicrob Agents Chemother. 2002;46(8):2640-3.

35. Mallea M, Mahamoud A, Chevalier J, Alibert-Franco S, Brouant P, Barbe J, et al. Alkylaminoquinolines inhibit the bacterial antibiotic efflux pump in multidrug-resistant clinical isolates. Biochem J. 2003;376(Pt 3):801-5.

36. Tran QT, Mahendran KR, Hajjar E, Ceccarelli M, Davin-Regli A, Winterhalter M, et al. Implication of porins in beta-lactam resistance of Providencia stuartii. J Biol Chem. 2010;285(42):32273-81.

37. http://www.cancer.org/acs/groups/content/@epidemiologysurveilance/ documents/document/acspc-031574.pdf. Cancer in Africa. Accessed on June12, 2012

38. Ichimura T, Yamanaka A, Ichiba T, Toyokawa T, Kamada Y, Tamamura T, et al Antihypertensive effect of an extract of Passiflora edulis rind in spontaneously hypertensive rats. Biosci Biotechnol Biochem. 2006;70:718-21.

39. Silva JR, Campos AC, Ferreira LM, Aranha Junior AA, Thiede A, Zago Filho LA, et al. [Extract of Passiflora edulis in the healing process of gastric sutures in rats: a morphological and tensiometric study]. Acta Cir Bras. 2006;21 Suppl 2:52-60.

40. Kannan S, Parimala B, Jayakar B. Antibacterial evaluation of the methanolic extract of Passiflora edulis. Hygeia JDMed. 2011;3(1):46-9.

41. Fuglie L. The miracle tree Moringa oleifera: natural nutrition for the tropics. Dakar: Church World Service; 1999.

42. Abrams B, Duncan D, Hertz-Picciotto I. A prospective study of dietary intake and acquired immune deficiency syndrome in HIV-seropositive homosexual men. J Acquir Immune Defic Syndr. 1993:6:949-58.

43. Fahey J. Moringa oleifera: A review of the medical evidence for its nutritional, therapeutic and prophylactic properties. Trees for Life Journal. 2005; $1: 1-15$

44. Viera GH, Mourao JA, Angelo AM, Costa RA, Vieira RH. Antibacterial effect (in vitro) of Moringa oleifera and Annona muricata against Gram positive and Gram negative bacteria. Rev Inst Med Trop Sao Paulo. 2010:52:129-32.

\section{Submit your next manuscript to BioMed Central and we will help you at every step:}

- We accept pre-submission inquiries

- Our selector tool helps you to find the most relevant journal

- We provide round the clock customer support

- Convenient online submission

- Thorough peer review

- Inclusion in PubMed and all major indexing services

- Maximum visibility for your research

Submit your manuscript at www.biomedcentral.com/submit 